\title{
Comparison of the relative transparency of the atmosphere measured by attenuation of Cherenkov light
}

\author{
Igor Petrov ${ }^{* \dagger}$ \\ Yu.G. Shafer Institute of Cosmophysical Research and Aeronomy \\ E-mail: igor.petrov@ikfia.ysn.ru

\section{Stanislav Knurenko} \\ Yu.G. Shafer Institute of Cosmophysical Research and Aeronomy \\ E-mail: knurenko@ikfia.ysn.ru
}

\begin{abstract}
A method for observing the state of the atmosphere during optical observations of VavilovCherenkov radiation in the region of the Yakutsk complex array is described. Classification of weather conditions during seasonal observations of the atmosphere (autumn-winter-spring) is given, using cosmic rays with energies (1-10) PeV. Empirical results are compared with calculations for different grades of visibility range. It is shown how the transparency of the atmosphere can influence the estimated of some characteristics of extensive air showers.
\end{abstract}

35th International Cosmic Ray Conference - ICRC2017-

12-20 July, 2017

Bexco, Busan, Korea

* Speaker.

${ }^{\dagger}$ A footnote may follow. 


\section{Introduction}

An important characteristic of extensive air showers (EAS) is the Vavilov-Cherenkov light, which is measured at the Yakutsk array by a system of optical detectors. For example, the total flux of Cherenkov light EAS is calculated at the observation level according to (1.1):

$$
\Phi\left(E_{0}\right)=4 \int_{E_{\min }}^{E_{0}} \tau \int^{X_{0}} \tau P\left(E_{g}, x\right) d E_{\gamma} d X
$$

Where $\mathrm{P}\left(\mathrm{E}_{\gamma}, \mathrm{x}\right)$ is the total flux of Cherenkov light in partial electron-photon avalanches and the density of the Cherenkov light flux at a distance of $400 \mathrm{~m}$ from the shower axis $\mathrm{Q}(\mathrm{R}=400)$, serve as the basis for the method of determining the energy of the primary particle generating the shower $[1,2]$. Because photons come from different altitudes of the atmosphere, the differential flux of Cherenkov light contains information on the longitudinal development of the cascade of relativistic particles $[3,4]$. Namely, the depth of the maximum of the development of the $X_{\max }$ shower, which is related to the atomic weight of the cosmic ray particles. All this is used as the basis for the development of techniques and further evaluation of the mass composition of cosmic rays of ultrahigh energies [5].

Therefore, in order to obtain true EAS characteristics, it is necessary to know the transmission function of Cherenkov light by the atmosphere during the entire observation period. ThatâÁŹs why at the Yakutsk array the state of the night sky has been monitored, for more than 40 years [6].

\section{Measurement of atmospheric transparency using EAS Cherenkov light at low energies.}

In the first approximation, air shower Cherenkov light can be characterized as a source of point light, which is at the height of the maximum development $\mathrm{X}_{\max }$ of the air shower. Usually, for showers with an energy of $10^{15}-10^{16} \mathrm{eV}$, the maximum development of the shower is in the upper layers of the troposphere. By registering the frequency of "flares" of such air showers, it is possible to plot a cosmic ray spectrum above given energy $\mathrm{E}_{0}$. It was shown in [7] that the shape of the spectrum with energies of $10^{15}-10^{16} \mathrm{eV}$ can be used to control the atmospheric transmission function, i.e. its transparency. This method evaluates the relative transparency to the most favorable atmospheric conditions, when the scattering and absorption of light is minimal. In this case, the transmittance can be determined by the formula:

$$
\tau_{r} e l=\tau_{0}\left(\frac{N}{N_{0}}\right)^{\frac{1}{k}}
$$

Where $\tau_{\text {rel }}$ is the transmittance of light by the atmosphere at the time of measurement, $\tau_{0}$ is the periods when the atmosphere is the cleanest, and $\mathrm{k}=1.7$ is the integral energy spectrum of the $\mathrm{CR}$ in the $10^{15} \mathrm{eV}$ region. The relation follows from the fact that the number of Cherenkov photons is proportional to the energy of the primary EAS particle. It is assumed that the absolute maximum of the atmospheric transparency $\tau_{0}$ corresponds to the minimum of the aerosol content. Such transparency of the atmosphere happens in the course of $\sim 5 \%$ of the total time of registration of Cherenkov radiation at the Yakutsk array. 
The results of measurements of the distribution of aerosols $[8,9]$ showed that above $1.5 \mathrm{~km}$ their content in the atmosphere is almost constant (the photon absorption length is $\Lambda_{M} \sim 200 \mathrm{~km}$ ), and below is approximately proportional to the air density. Using the linear approximation of the altitude data for $\mathrm{h}<1.5 \mathrm{~km}$ and a constant $\Lambda_{M}$ above $1.5 \mathrm{~km}$, we obtained the light transmittance shown in Fig. 1, when light was emitted at a depth of $\mathrm{X}_{\max }$. The aerosol concentration in the surface air layer in this case was chosen so that the total transmission coefficient corresponded to the measurements in the area of the Yakutsk array. For comparison, the values calculated by formula (2.2) are also given here:

$$
\tau=0.522+0.24 X_{\max } / 1000
$$

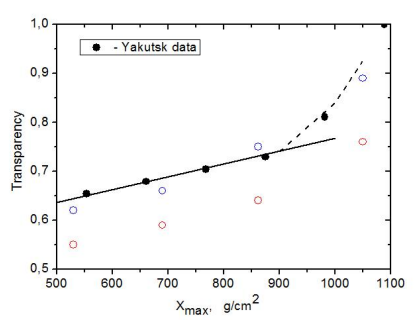

Figure 1: Dependence of light transmittance in the atmosphere on depth of the maximum at $\theta=20^{\circ}$. Dark circles - estimation of the transparency taking into account the distribution of aerosol density, straight formula (1), dashes - formula (2). Blue and red circles - the result of atmospheric transparency reconstruction by Cherenkov light observation, registered at different weather conditions, 5 and 4 points respectively.

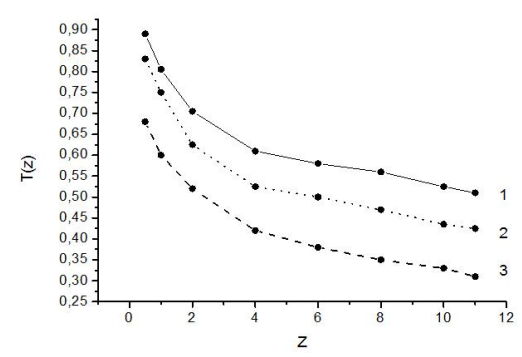

Figure 2: Vertical profile of atmospheric transmittance at different conditions: 1 - 5 points; 2 - 4 points; $3-3$ points.

which was obtained without taking into account non-uniformity of the aerosol concentration in the atmosphere during the winter at the Yakutsk. It can be seen that the approach used previously did not fits to the measurements [6]. Mainly due to incorrect altitude depending on the concentration of aerosols in the atmospheric boundary layer.

The corrected value of the total light transmittance can be represented by the expression for the altitude region $\mathrm{h}<1.5 \sec \theta \mathrm{km}$ :

$$
\tau=4.1-8 \times 10^{-3} X_{\max }+\frac{X_{\max }^{2}}{2.11 \times 10^{5}}
$$

where $\mathrm{X}_{\max }$ in $\mathrm{g} / \mathrm{cm}^{2}$.

\section{Loss of light depending on the thickness of the atmosphere}

At present, using the experimental Cherenkov light LDF (CLLDF) with different transparency of the atmosphere and the method of solving the inverse problem, we have restored the altitude 
variation of the light attenuation coefficient [10]. The result is shown in Fig.2. It is seen that for different optical states of the atmosphere, there is a difference both in absolute value at the observation level and in the vertical profile of the atmospheric transmittance. The greatest contribution to the transmittance of the atmosphere is made by the aerosol component of the surface layer of the atmosphere, i.e. height $\sim 2,4 \mathrm{~km}$ from sea level. According to [10], the fraction of the aerosol component is more significant for the transparency of the atmosphere with a visual estimate of three points. Usually such periods of observation were not included in the analysis. The course of the atmospheric transmittance as a function of altitude for the most excellent optical conditions for observing Cherenkov radiation can be approximated by the following expression:

$T(z)=\exp \left\{-\left[0.3757+0.0383 \cdot \exp \left(-\frac{h}{h_{0}}\right)\right] \cdot\left[1-\exp \left(-\frac{h}{h_{0}}\right)\right]-0.2933\left[1-\exp \left(-\frac{h}{h_{A}}\right)\right]\right\}$

where $\mathrm{h}_{0}=6800 \mathrm{~m}, \mathrm{~h}=1500 \mathrm{~m}$.

The expression (3.1) was subsequently used in calculation the expected functions of the spatial distribution of the Cherenkov light.

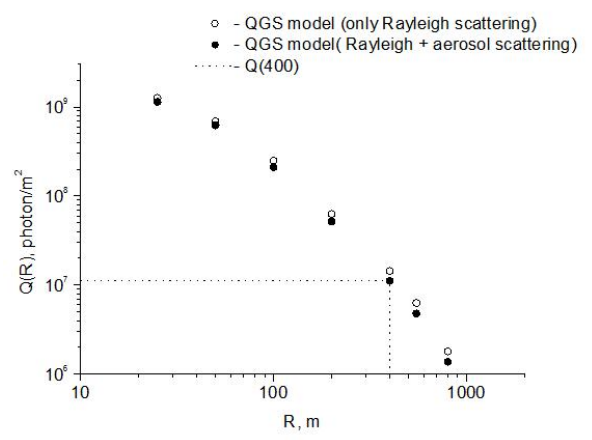

Figure 3: CLLDF determination at different atmospheric conditions. In the calculation, the actual transmission of the atmosphere over the Yakutsk array in winter is taken. The case of 5 and 3 points is considered.

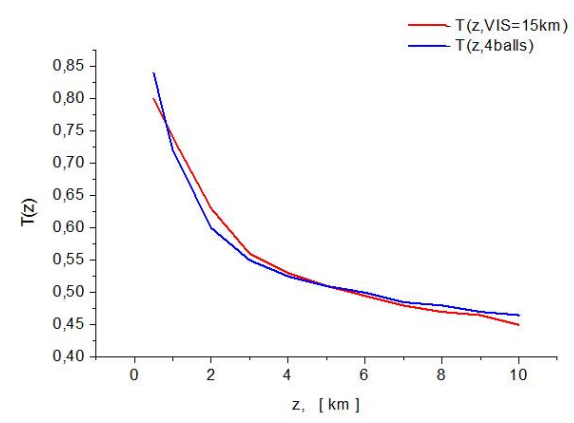

Figure 4: The transmission coefficients for the atmosphere are 4 points and for the visibility range parameter $\mathrm{V}=15 \mathrm{~km}$

From Fig.3. It can be seen that in the presence of an aerosol in the atmosphere, the shape of the Cherenkov light function varies, and especially at large distances from the shower axis. This 
circumstance leads to an increase in the total flux of Cherenkov light EAS by (10 - 15)\%, which automatically leads to an overestimation of the shower energy, which is determined using this characteristic. Taking into account the effect of the atmospheric transmittance when the cascade development curve of the EAS is reconstructed from the spatial distribution of the Cerenkov light, EAS gives the correct value of $\mathrm{X}_{\max }$.

\section{Conclusion}

Thus, taking into account the losses of the Cherenkov light photons in the atmosphere becomes necessary when obtaining qualitative data on cosmic radiation. In Fig. 4, a comparison of the empirically obtained profile of the atmospheric transmittance function at 4 points according to the classification [11], with the calculated value obtained with an apparent range of $\mathrm{V}=15 \mathrm{~km}$ [12]. The comparison showed good agreement of the results. This indicates the competence of using in the first approximation a visual assessment of transparency for rapid assessment of atmospheric conditions for the purpose of conducting patrol optical observations.

\section{Acknowledgments}

The reported study was funded by RFBR according to the research project 16-29-13019.

\section{References}

[1] S.P. Knurenko, A.A. Ivanov, I.Ye. Sleptsov and A.V. Sabourov, JETP Lett. V. 83, No. 11, 563-567 (2006).

[2] A.A. Ivanov, S.P. Knurenko, M.I. Pravdin, I.Ye. Sleptsov, JETP, v. 131, No. 6, 1001-1017 (2007).

[3] Dyakonov M.N.,Knurenko S.P., Kolosov V.A., Krasilnikov D.D. et al. The use of Cherenkov detectors at the Yakutsk cosmic ray extensive Đřir shower array. //Nuclear Instruments and Meth-s in Phys. Rec. Amst., A248, v.4, p. 224-226 (1986).

[4] S.P. Knurenko et al. Proc. 27th ICRC. Hamburg. 1, 157 (2001).

[5] Stanislav Knurenko and Igor Petrov. Mass Composition of Cosmic Rays at Ultra High Energies by Yakutsk Data. //2015 J. Phys.: Conf. Ser. 632012098 doi:10.1088/1742-6596/632/1/012098.

[6] S.P. Knurenko, I.S. Petrov. XIX Intern. Symp. "Optics of the atmosphere and ocean. Physics of the atmosphere", D357-D360 (2013).

[7] M.N. Dyakonov, S.P. Knurenko, V.A. Kolosov, I.Ye. Sleptsov. Optics of the atmosphere, V. 4, p.868-873 (1991).

[8] M.A. Mostafa et al. (Pierre Auger collaboration). Atmospheric monitoring for the Pierre Auger fluorescence detector. // Proc. 28th ICRC, Tsukuba. 2003. Vol. 1. P. 465 - 468.

[9] http: // aeronet.gshc.nasa.gov./

[10] M.N. Dyakonov, S.P. Knurenko, V.A. Kolosov, I.Ye. Sleptsov. Optics of the atmosphere and ocean, V. 12, No. 4, p.329-333 (1999).

[11] S.P. Knurenko, S.V. Nikolashkin, A.V. Saburov, I.Ye. Sleptsov. Study of atmosphere characteristics using ultra-high energy cosmic ray and ïAn̆ $=532 \mathrm{~nm}$ LIDAR. // Proc. Of SPIE. Vol. 6522 (2006).

[12] A.I. Goncharov, N.G. Nikitenko. Bulletin of Altai State University, No.5, p. 85-88 (2004). 